\section{BRAZIULIAN JOURNAL}

OF MEDICAL AND BIOLOGICAL RESFARCH

www.bjournal.com.br
ISSN 0100-879X

Volume 43 (7) 600-697 July 2010

BIOMEDICAL SCIENCES

AND

CLINICAL INVESTIGATION

Braz J Med Biol Res, July 2010, Volume 43(7) 627-633

doi: 10.1590/S0100-879X2010007500043

Quantitative evaluation of experimental choroidal neovascularization by confocal scanning laser ophthalmoscopy. Fluorescein angiogram parallels heparan sulfate proteoglycan expression

C.V. Regatieri, J.L. Dreyfuss, G.B. Melo, D. Lavinsky, S.K. Hossaka, E.B. Rodrigues, M.E. Farah, M. Maia and H.B. Nader

The Brazilian Journal of Medical and Biological Research is partially financed by

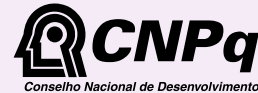

Ministério

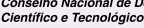

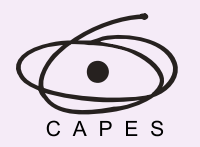

Ministério da Educação

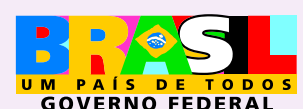

GOVERNO FEDERAL

\section{JAAPESP}

Institutional Sponsors
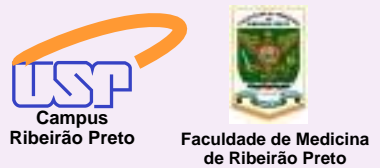

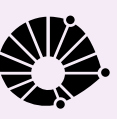

UNICAMP $\oplus$ SHIMADZU

GE Healthcare
Hotsite of proteomics metabolomics developped by:

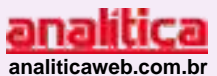

Thermo
SCIEN TIFIC 


\title{
Quantitative evaluation of experimental choroidal neovascularization by confocal scanning laser ophthalmoscopy. Fluorescein angiogram parallels heparan sulfate proteoglycan expression
}

\author{
C.V. Regatieri, ${ }^{1,2}$, J.L. Dreyfuss ${ }^{2}$, G.B. Melo ${ }^{1}$, D. Lavinsky ${ }^{1}$, \\ S.K. Hossaka ${ }^{1}$, E.B. Rodrigues ${ }^{1}$, M.E. Farah ${ }^{1}$, M. Maia ${ }^{1}$ and H.B. Nader ${ }^{2}$ \\ ${ }^{1}$ Departamento de Oftalmologia, ${ }^{2}$ Departamento de Bioquímica, \\ Universidade Federal de São Paulo, São Paulo, SP, Brasil
}

\begin{abstract}
The objective of the present study was to develop a quantitative method to evaluate laser-induced choroidal neovascularization (CNV) in a rat model using Heidelberg Retina Angiograph 2 (HRA2) imaging. The expression of two heparan sulfate proteoglycans (HSPG) related to inflammation and angiogenesis was also investigated. CNV lesions were induced with argon laser in 21 heterozygous Zucker rats and after three weeks a fluorescein angiogram and autofluorescence exams were performed using HRA2. The area and greatest linear dimension were measured by two observers not aware of the protocol. Bland-Altman plots showed agreement between the observers, suggesting that the technique was reproducible. After fluorescein angiogram, HSPG (perlecan and syndecan-4) were analyzed by real-time RT-PCR and immunohistochemistry. There was a significant increase in the expression of perlecan and syndecan-4 $(P<0.0001)$ in retinas bearing CNV lesions compared to control retinas. The expression of these two HSPG increased with increasing CNV area. Immunohistochemistry demonstrated that the rat retina damaged with laser shots presented increased expression of perlecan and syndecan-4. Moreover, we observed that the overexpression occurred in the outer layer of the retina, which is related to choroidal damage. It was possible to develop a standardized quantitative method to evaluate $\mathrm{CNV}$ in a rat model using HRA2. In addition, we presented data indicating that the expression of HSPG parallels the area of CNV lesion. The understanding of these events offers opportunities for studies of new therapeutic interventions targeting these HSPG.
\end{abstract}

Key words: Choroidal neovascularization; Fluorescein angiogram; Proteoglycans; Angiogenesis; Confocal scanning laser ophthalmoscope

\section{Introduction}

Age-related macular degeneration associated with choroidal neovascularization (CNV) is a major cause of severe visual loss in western countries (1). The newly formed vessels, originating from the choroid, grow into the subretinal space, where they are recognized clinically by fluorescence leakage during a fluorescein angiogram (FA) (2). CNV may also be a common finding in many other eye diseases, such as pathological myopia, angioid streaks and presumed ocular histoplasmosis syndrome.

In 1979, Ryan (3) developed the first animal model for
CNV using Rhesus monkeys and argon laser photocoagulation to damage Bruch's membrane. Since then, other models have been developed in mice, rats and pigs using argon and diode lasers, mechanical stress, enzymatic digestion of Bruch's membrane, transchoroidal infusion of fibroblast growth factor-2 (FGF-2) or vascular endothelial growth factor (VEGF) (4-7). A current reproducible animal model for CNV studies is the choroidal damage induced by argon or diode laser in mice and rats (8-10).

Quantitative assessment of retinal and choroidal morphol-

Correspondence: H.B. Nader, Disciplina de Biologia Molecular, UNIFESP, Rua Três de Maio, 100, 04044-020 São Paulo, SP, Brasil. Fax: +55-11-5573-6407. E-mail: hbnader.bioq@epm.br

Received January 7, 2010. Accepted April 28, 2010. Available online May 14, 2010. Published July 9, 2010. 
ogy is a critical step in the study of the physiopathology of neovascular disease, and is used to monitor disease progression and to evaluate the effects of drugs on CNV inhibition.

The Heidelberg Retina Angiograph 2 (HRA2) is a digital confocal scanning laser ophthalmoscope (cSLO) used for fundus imaging. It is based on the property of scanning the fundus with a laser beam from a point source, while the reflected light is detected by a photomultiplier detector. Incident and reflected lights follow a coaxial path (11).

Several studies have described the use of fundus imaging in rodent models for a variety of diseases (11-16). However, the methods published thus far do not allow precise quantitative measurements of CNV.

Angiogenesis is an intricate process that involves many steps including a myriad of angiogenic agents as growth factors, limited proteolysis mediated by specific metalloproteinases and the interaction of components of the extracellular matrix (ECM) with their receptors (17-19). Heparan sulfate proteoglycans (HSPG) have been shown to be involved in the modulation of neovascularization, and are ubiquitously found on the cell surface and in the ECM of all animal species $(20,21)$. The heparan sulfate chains, due to their vast structural diversity, are able to bind and interact with a wide variety of proteins, such as growth factors, chemokines, morphogens, ECM components, enzymes, and others $(22,23)$. Among the cell surface HSPG, syndecan-4 is always present in endothelial cells. The heparan sulfate chains of syndecan- 4 are considered to play various roles by binding and modulating growth factors such as FGF-2, VEGF, tumor growth factor- $\beta$ (TGF- $\beta$ ), anticoagulation factors, such as tissue factor pathway inhibitors, and cell adhesion molecules $(24,25)$. These interactions trigger signaling pathways either directly by the phosphorylation of cytoplasmic proteins or via the cytoskeleton by the use of the cytoplasmic domain of the core protein (26). Furthermore, syndecan-4 also contributes to endothelial tubulogenesis (27).

Perlecan is a basement membrane HSPG, which can bind several growth factors such as FGF, VEGF, and PDGF, among others (28). Perlecan not only has structural roles in the molecular assembly and maintenance of basement membranes, but can also play an instructive role by providing information to integrins and to other surface receptors by acting as co-receptors and growth factor scavengers. All of these biological activities undoubtedly influence the proliferation, migration and morphogenesis of endothelial cells $(29,30)$. HSPG play an important role in inflammation since there is evidence that these molecules interact with L-selectin and chemokines $(21,31)$.

The present study was designed to develop a quantitative method to evaluate laser-induced $\mathrm{CNV}$ in a rat model using HRA2. Since HSPG are involved in inflammation and angiogenesis, the expression of perlecan and syndecan-4 in retina was also investigated by real-time RT-PCR and immunohistochemistry.

\section{Material and Methods}

\section{Animals}

All experiments were performed in accordance with the Association for Research in Vision and Ophthalmology Statement for Use of Animals in Ophthalmic and Vision Research, and in accordance with a protocol reviewed and approved by the Animal Care Committee of the Federal University of São Paulo (\#1388/06). The study was carried out on 21 female heterozygous pigmented Zucker rats weighing 180 to $220 \mathrm{~g}$.

\section{Induction of choroidal neovascularization}

Animals were anesthetized with an intramuscular injection of a mixture of $56 \mathrm{mg} / \mathrm{kg}$ ketamine and $6 \mathrm{mg} / \mathrm{kg}$ xylazine. The pupil was then dilated by topical application of a combination of $1 \%$ tropicamide and $2.5 \%$ phenylephrine hydrochloride (Allergan, Brazil). Photocoagulation was performed using an argon laser $(532 \mu \mathrm{m})$ connected to a slit lamp delivery system (SL 115 Slit Lamp, Carl Zeiss Meditec, Germany). A hand-held coverslip was used as a contact lens by direct contact with the cornea of the animals using $1 \%$ methyl cellulose (Ophthalmos, Brazil). Four lesions located at the $3,6,9$, and 12 o'clock meridians, centered on the optic nerve head and located approximately 2 disc diameters from the head of the optic nerve, were created using a power of $300 \mathrm{~mW}$, spot size of $100 \mu \mathrm{m}$ and duration of $100 \mathrm{~ms}$. The objective was to create a rupture in Bruch's membrane, confirmed by bubble formation, to allow the invasion of the subretinal space by choroidal blood vessels, resulting in a neovascular membrane. This procedure was performed in the right eye of 21 animals, and the left eye was used as control.

\section{Fluorescein angiogram}

Three weeks after CNV induction, animals were anesthetized and pupils dilated as described above. FA was performed using an HRA2 instrument (Heidelberg Engineering, Germany) (11). The highest definition image was 768 x 768 pixels. CNV lesion was measured using a $55^{\circ}$ lens with the same focus in all cases. Autofluorescence is a property of fundus image without the use of any fluorescent substance when it is excited with a 488-nm wavelength laser. The images were obtained with HRA2 (32) before fluorescein injection. Rats were manually held in front of HRA2 device, in an upright position and FA was performed. Fluorescein injections were administered intraperitoneally (0.2 mL 2\% fluorescein; Ophthalmos), and the timer was started as soon as the fluorescein bolus was injected. All early images were captured immediately after fluorescein injection and all late phase images 12 min after the injections. During the exams the corneas were keep moist with $2 \%$ methylcellulose (Ophthalmos). Twenty-one days after laser induction of CNV, two observers with experience in FA evaluation and not involved in the experiments assessed 
the FA. The image of the fluorescein leakage area in the late phase was reported in square millimeters $\left(\mathrm{mm}^{2}\right)$ using HRA2 software. The image of the greatest linear diameter was also measured in millimeters $(\mathrm{mm})$.

\section{Retinal RNA extraction and gene expression analysis}

After 3 weeks, eyes from three animals ( 6 eyes, 3 control eyes and 3 with laser-induced CNV: rat 1 , rat 2 and rat 3 ) were enucleated and the retina was removed for RNA extraction. Detection of mRNAs encoding syndecan-4, perlecan and $\beta$-actin (as endogenous control) was analyzed by quantitative RT-PCR. Total retinal tissue RNA was isolated using Trizol reagent (Gibco BRL, USA), followed by the synthesis of cDNA by reverse transcription using the IMPRON II kit (Promega, USA). The sets of primers used were as follows: perlecan: sense 5'-CTGCCACCTG ACAGTCGC-3' and antisense 5'-GCTCTGGCACCTGC AG-3' (gene data bank accession number NM 005529.5); syndecan-4: sense 5'-GGGCAGCAACATCTTTGAGAG-3' and antisense 5'-TCCTTCTTCTTCATACGGTAC-3' (gene data bank accession number NM 002999.2); $\beta$-actin: sense 5'-CATCCTGCGTCTGGACCTGG-3' and antisense: 5'-GGCAGCTCATAGCTCTTC-3' (gene data bank accession number NM 001101.3).

Is important to point out that are similarities between Rattus novergicus and Homo sapiens in these HSPG genes. Real-time PCR was performed using a 7500 real-time PCR System (Applied Biosystems, USA) and the reactions were performed using SYBR Green PCR Master Mix reagents (Applied Biosystems). Temperatures were set at $55^{\circ} \mathrm{C}$ for annealing, $72^{\circ} \mathrm{C}$ for extension and $94^{\circ} \mathrm{C}$ for denaturation for 45 cycles for all genes analyzed. The experiments were performed in hexaplicate and data are reported as means \pm SEM.

\section{Quantification and localization of HSPGs by immunohistochemistry}

Formaldehyde-fixed paraffin-embedded eyes submitted or not to laser shots were investigated. After clearing with xylene, the slides were dehydrated in alcoholic solutions, treated with $3 \% \mathrm{H}_{2} \mathrm{O}_{2}$ for 35 min to inhibit endogenous peroxidase activity, and blocked with $1 \%$ bovine serum albumin for $1 \mathrm{~h}$. The slides were then incubated overnight with primary antibodies: a mouse monoclonal anti-syndecan 4 (4E12A8 clone) raised against the $29 \mathrm{~N}$-terminal portion of the syndecan-4 protein and a polyclonal anti-perlecan (Santa Cruz, USA). After washing in PBS, sections were incubated with the universal LSAB kit (Dako, Denmark), washed in PBS and incubated with a liquid diaminobenzidine substrate (10 $\mathrm{s}$; Dako). The slides were finally counterstained with hematoxylin and analyzed by light microscopy (Nikon Eclipse E600, objective 20 or $40 \mathrm{X}$ ).

\section{Statistical analysis}

To assess interobserver reproducibility, leakage area and greatest linear dimension were compared for the images acquired by observer 1 and observer 2 using Bland-Altman plots (33). The reproducibility of these features was quantified using the bias (mean difference) and analyzed by the concordance coefficient (34) with a 95\% confidence interval. The minimum required sample is 13 animals assuming a sampling error of $10 \mathrm{~mm}$ for greatest linear diameter and a $95 \%$ confidence (34). Data are reported as means \pm SEM for real-time PCR analysis. Statistical analyses were performed by one-way ANOVA with Bonferroni's post-test using the Graph Pad Prism 5.0 software for Windows. A 95\% confidence interval and a $5 \%$ level of significance were adopted. $P$ values $\leq 0.05$ were considered to be significant.

\section{Results}

\section{Fluorescein angiogram analysis}

Three weeks after laser induction, 73 of 84 lesions (86.9\%) presented clinically significant leakage by FA. Two eyes presented cataract formation, but FA could still be performed. Satisfactory fundus imaging was obtained in all exams. The exams were performed easily without any complementary lens.

The measurement of the greatest linear diameter as well as the leakage area reported by different examiners was statistically similar, indicating interexaminer reproducibility. Both observers easily measured the entire leakage area and greatest linear diameter using the HRA software. The mean area reported by examiner 1 was $1909 \pm 324$ $\mathrm{mm}^{2}$ and the mean area reported by examiner 2 was 2115 $\pm 333 \mathrm{~mm}^{2}$. Examiner 1 reported a mean greatest linear diameter of $58.16 \pm 4.6 \mathrm{~mm}$ and examiner 2 reported a value of $55.8 \pm 5.0 \mathrm{~mm}$ based on same images. A Bland-Altman plot showed good interexaminer agreement for measuring greatest linear diameter, suggesting that this technique is reproducible. The mean difference in the measurement of greatest linear diameter between the two examiners was 0.4 with limits of agreement of $95 \%(-29.4 ;+30.1)$ and the values were equally distributed around negative and positive differences (Figure 1). Good concordance coefficients for both greatest linear diameter and area were found $(0.624$ and 0.606 , respectively). The concordance interval was $0.231-0.844$ with $P=0.002$ for greatest linear diameter and 0.204-0.836 with $P=0.003$ for area.

The lesion shape observed in the FA was similar in all cases and the intensity of leakage was sufficient to determine the area in the late phase. Figure $2 \mathrm{~A}$ shows the control eye and Figure 2B,C shows FA after 21 days of laser-induced CNV, illustrating the circulatory filling of the vessels. The autofluorescence exam using the 488-nm laser did not present any hyperautofluorescent points in the control eye (Figure 2D). On the other hand, in animals submitted to laser shots, hyperautofluorescent points were observed surrounding the CNV lesions (Figure 2E,F). Autofluorescence was present in $50 \%$ of CNV lesions and was 
not related to lesion size or serous retinal detachment.

\section{Expression of heparan sulfate proteoglycans in the retina}

The expression of syndecan-4, a typical endothelial cell surface proteoglycan, and perlecan, a typical endothelial cell ECM, was analyzed by real-time RT-PCR in three rat retinas (rat 1 , rat 2 and rat 3 ). There was a significant increase $(P<0.0001)$ in the expression of both perlecan and syndecan 4 in retinas bearing $\mathrm{CNV}$ lesions compared to control retinas (Figure 3 ). The expression of these two
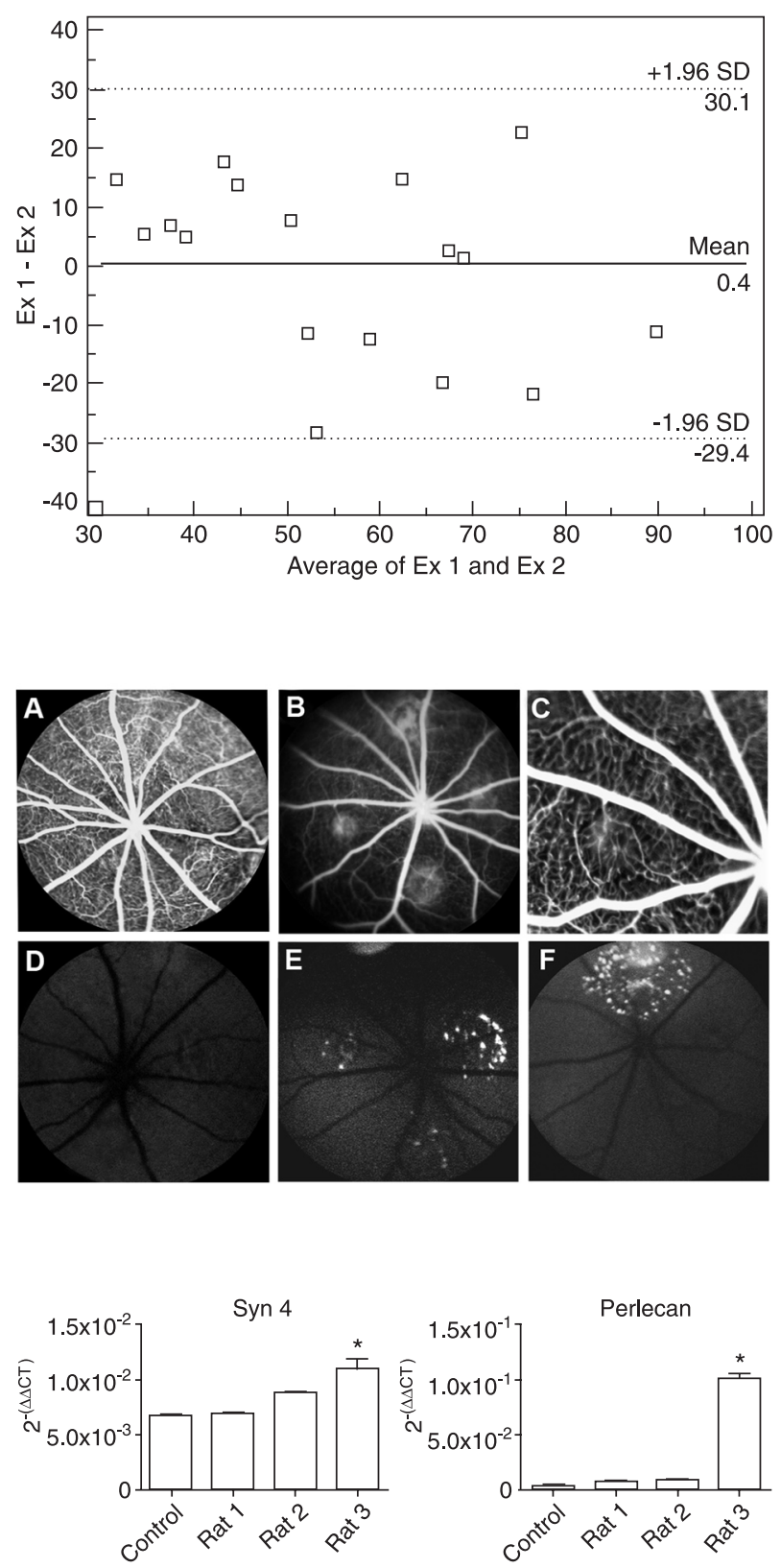

HSPG increased with increasing CNV lesion area. The sum of the areas of $4 \mathrm{CNV}$ lesions was rat $1=7,583$, rat 2 $=9,184$, and rat $3=28,038 \mathrm{~mm}^{2}$.

Immunohistochemical analysis of HSPG in the retina

Syndecan-4 and perlecan expression in rat retina with and without laser treatment was also investigated by immunohistochemistry. Retinas submitted to laser shots showed increased expression of both syndecan-4 (Figure 4) and perlecan (Figure 5) compared to control retinas. An increase in the expression of syndecan-4 located on the cell surface

Figure 1. Bland-Altman plot of greatest linear diameter measurement by two examiners (Ex 1, Ex 2) [mean difference, 0.4; limits of agreement of $95 \%(-29.4 ;+30.1)]$.

Figure 2. Fluorescein angiogram and autofluorescence exam with HRA2 after three weeks of laser-induced choroidal neovascularization (CNV). Panels $A, B$ and $C$, Fluorescein angiogram in the same eye: control eye (A); initial phase using a $55^{\circ}$ lens with zoom captured immediately after fluorescein injection (B); late phase acquired with a $55^{\circ}$ lens captured 12 min after the fluorescein injections (C). Panels $D, E$ and $F$, Autofluorescence exam acquired with a $55^{\circ}$ lens: control eye (D); autofluorescence exam of two different eyes submitted to CNV $(E, F)$.

Figure 3. Expression of syndecan 4 and perlecan in retinas submitted to laser-induced experimental choroidal neovascularization (CNV). Expression was measured by quantitative RTPCR. The histograms show the relative expression of syndecan 4 (Syn 4) or perlecan with respect to $\beta$-actin. Control = retinas without laser treatment; rat 1,2 , and $3=$ experimental group submitted to CNV. ${ }^{*} P<0.0001$ compared to other groups (ANOVA with Bonferroni's post-test). 
of the outer nuclear layer and photoreceptor segment layers can be seen after the laser shots. Similar results were obtained for perlecan, which was overexpressed in the
ECM of the outer nuclear layer and photoreceptor segment layers after the laser shots.

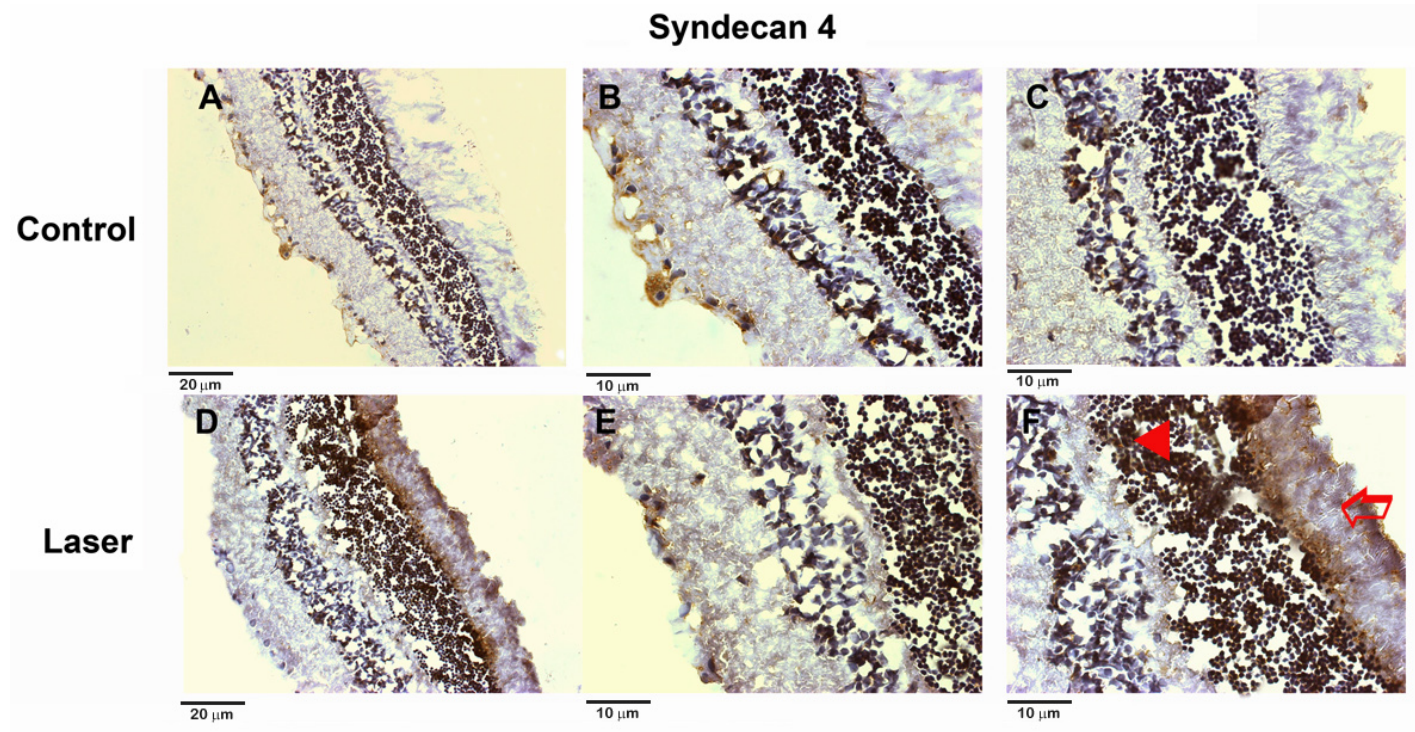

Figure 4. Immunohistochemical analysis of syndecan 4 in retinas after laser shots. The upper panels show a control retina. $A, 20 \mathrm{X}$ magnification; $B, C, 40 \mathrm{X}$ magnification. The lower panels show a retina submitted to laser shots, the immunohistochemical staining is showed in brown. $D, 20 \mathrm{X}$ magnification; $E, F, 40 \mathrm{X}$ magnification. The arrowhead indicates the brown staining of syndecan-4 in the outer nuclear layer and the open arrow indicates the brown staining of syndecan- 4 in the photoreceptor segment layer.
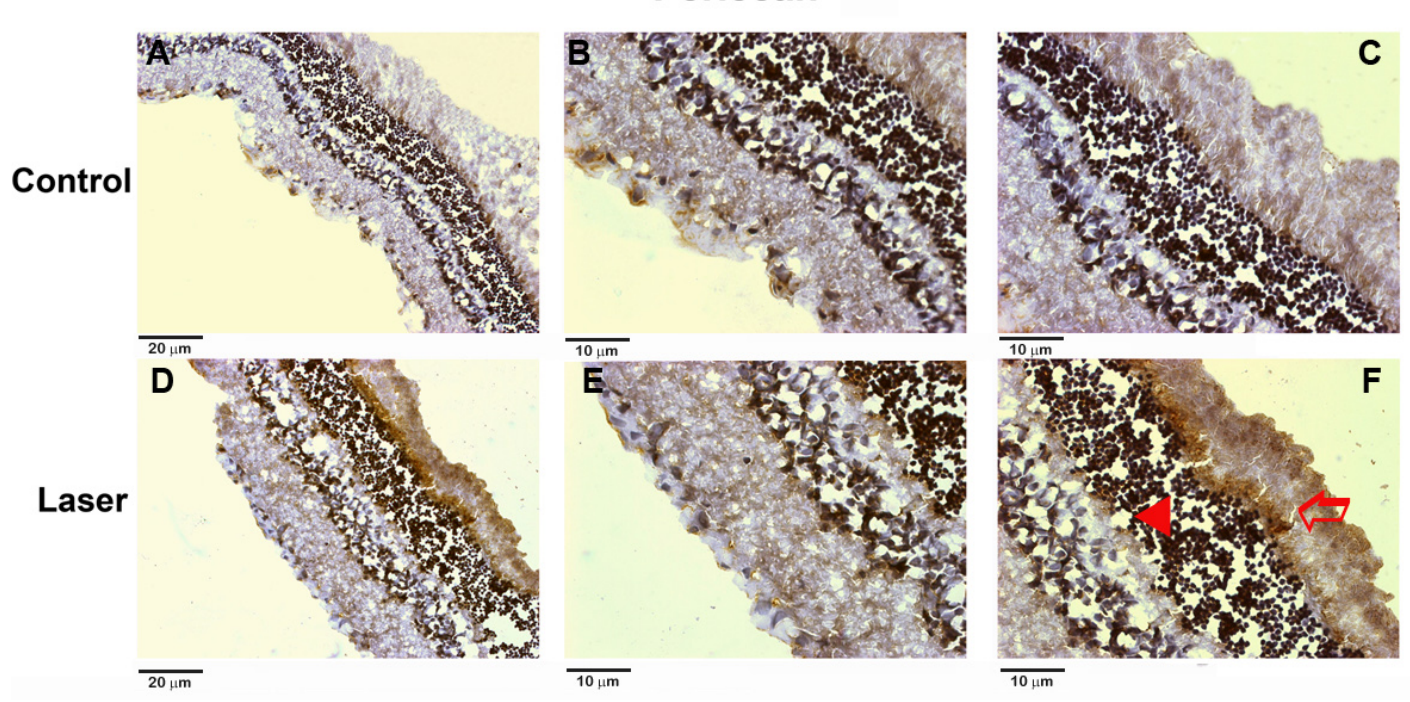

Figure 5. Immunohistochemical analysis of perlecan in retinas after laser shots. The upper panels show a control retina. $A, 20 \mathrm{X}$ magnification; $B, C, 40 \mathrm{X}$ magnification. The lower panels show a retina submitted to laser shots. $D$, 20X magnification; $E, F, 40 \mathrm{X}$ magnification. The arrowhead indicates the brown staining of perlecan in the outer nuclear layer and the open arrow indicates the brown staining of perlecan in the photoreceptor segment layer. 


\section{Discussion}

The objective of this study was to assess the capability of cSLO to visualize and measure CNV. cSLO appears to be a useful technique for experimental studies of vitreoretinal diseases, especially vascular disease models, and can also be used as a technique for in vivo cellular imaging of the retina $(11,35)$. Other current applications of cSLO include the study of neovascularization. In the present investigation, using cSLO we were able to develop a quantitative procedure to study neovascularization in an experimental CNV model. The entire CNV lesion was clearly and easily identified, corresponding to the leakage area. The measurements could be easily performed using software, and reproducibility was demonstrated by agreement of data obtained by two observers who were not involved in the experiments. In contrast to previous reports (9), the FA and autofluorescence exams were performed without any additional lenses. According to Paques et al. (11), FA has the disadvantage of a limited field of a single image, with the need to obtain a composite image. In the experiments described here using the 55-degrees HRA2 lens we were able to evaluate the $4 \mathrm{CNV}$ lesions in one image.

The in vivo autofluorescence of human retinal pigmented epithelium (RPE) provides a noninvasive technique that can be used in the investigation of inherited and age-related retinal diseases $(36,37)$, permitting the assessment of the viability of the RPE/photoreceptor complex by the distribution of the levels of retinal fluorophores (38). The ocular fundi of individuals with degenerative macular diseases such as age-related macular degeneration show a dramatic, agedependent accumulation of retinal fluorophores (38). Our data show that autofluorescence is preserved in animals without laser-induced $\mathrm{CNV}$, indicating normal function of the RPE/photoreceptor complex. In addition, the results obtained for the laser-induced CNV group were similar to those obtained for age-related macular degeneration, showing interesting images of hyperautofluorescent points close to the CNV lesion present in $50 \%$ of the lesions. We speculate that the hyperautofluorescent points are correlated with the presence of lipofuscin fluorophores, which emit light in the broad range of $500-750 \mathrm{~nm}$, indicating injury to the RPE/photoreceptor complex (39).

Changes in HSPG expression were observed in retinas submitted to laser-induced CNV. The increased expression of HSPG in CNV lesions may be related to endothelial dysfunction and increased capillary permeability. Our results showed that the major overexpressed HSPG was perlecan, that can serve as a scaffold for blood vessel formation (40), modulating the activities of angiogenic growth factors. Immunohistochemistry confirmed this increase of perlecan. Moreover, we observed that overexpression occurred on the outer retina layers, which are related to choroidal damage. Similar results were also observed for the immunohistochemical staining of syndecan-4, which may be related to the inflammatory process evoked by this experimental model. A previous study showed an increase of heparan sulfate synthesis in retinas submitted to laser shots by using metabolic radiolabeling with [ $\left.{ }^{35} \mathrm{~S}\right]$-sulfate in the same experimental model (21).

In summary, cSLO can be a useful and easy technique for the quantitative assessment of laser-induced CNV in a rat model. We presented data correlating the area of CNV lesion and the expression of heparan sulfate proteoglycans. The understanding of these events at the molecular level offers opportunities for new therapeutic interventions.

\section{Acknowledgments}

Research supported by FAPESP, CNPq, and CAPES.

\section{References}

1. Cui JZ, Kimura H, Spee C, Thumann G, Hinton DR, Ryan SJ. Natural history of choroidal neovascularization induced by vascular endothelial growth factor in the primate. Graefes Arch Clin Exp Ophthalmol 2000; 238: 326-333.

2. Teeters VW, Bird AC. The development of neovascularization of senile disciform macular degeneration. Am J Ophthalmol 1973; 76: 1-18.

3. Ryan SJ. The development of an experimental model of subretinal neovascularization in disciform macular degeneration. Trans Am Ophthalmol Soc 1979; 77: 707-745.

4. Campochiaro PA. Retinal and choroidal neovascularization. J Cell Physiol 2000; 184: 301-310.

5. Ryan SJ, Mittl RN, Maumenee AE. Enzymatic and mechanically induced subretinal neovascularization. Albrecht Von Graefes Arch Klin Exp Ophthalmol 1980; 215: 21-27.

6. Qiu G, Stewart JM, Sadda S, Freda R, Lee S, Guven D, et al. A new model of experimental subretinal neovascularization in the rabbit. Exp Eye Res 2006; 83: 141-152.
7. Wang $Y S$, Eichler W, Friedrichs $U$, Yafai $Y$, Hoffmann $S$, Yasukawa T, et al. Impact of endostatin on bFGF-induced proliferation, migration, and matrix metalloproteinase-2 expression/secretion of bovine choroidal endothelial cells. Curr Eye Res 2005; 30: 479-489.

8. Kvanta A, Shen WY, Sarman S, Seregard S, Steen B, Rakoczy E. Matrix metalloproteinase (MMP) expression in experimental choroidal neovascularization. Curr Eye Res 2000; 21: 684-690.

9. Shi X, Semkova I, Muther PS, Dell S, Kociok N, Joussen AM. Inhibition of TNF-alpha reduces laser-induced choroidal neovascularization. Exp Eye Res 2006; 83: 1325-1334.

10. Kohri T, Moriwaki M, Nakajima M, Tabuchi H, Shiraki K. Reduction of experimental laser-induced choroidal neovascularization by orally administered BPHA, a selective metalloproteinase inhibitor. Graefes Arch Clin Exp Ophthalmol 2003; 241: 943-952.

11. Paques M, Simonutti M, Roux MJ, Picaud S, Levavasseur E, 
Bellman C, et al. High resolution fundus imaging by confocal scanning laser ophthalmoscopy in the mouse. Vision Res 2006; 46: 1336-1345.

12. Murata $\mathrm{H}$, Aihara $\mathrm{M}$, Chen $\mathrm{YN}$, Ota T, Numaga J, Araie M. Imaging mouse retinal ganglion cells and their loss in vivo by a fundus camera in the normal and ischemia-reperfusion model. Invest Ophthalmol Vis Sci 2008; 49: 5546-5552.

13. Xu H, Koch P, Chen M, Lau A, Reid DM, Forrester JV. A clinical grading system for retinal inflammation in the chronic model of experimental autoimmune uveoretinitis using digital fundus images. Exp Eye Res 2008; 87: 319-326.

14. Goldenberg-Cohen N, Dadon S, Avraham BC, Kramer M, Hasanreisoglu M, Eldar I, et al. Molecular and histological changes following central retinal artery occlusion in a mouse model. Exp Eye Res 2008; 87: 327-333.

15. Falkenstein IA, Cheng L, Wong-Staal F, Tammewar AM, Barron EC, Silva GA, et al. Toxicity and intraocular properties of a novel long-acting anti-proliferative and anti-angiogenic compound IMS2186. Curr Eye Res 2008; 33: 599-609.

16. Julien S, Kreppel F, Beck S, Heiduschka P, Brito V, Schnichels $S$, et al. A reproducible and quantifiable model of choroidal neovascularization induced by VEGF A165 after subretinal adenoviral gene transfer in the rabbit. Mol Vis 2008; 14: 1358-1372.

17. Iozzo RV, San Antonio JD. Heparan sulfate proteoglycans: heavy hitters in the angiogenesis arena. J Clin Invest 2001; 108: 349-355.

18. Ishida S, Usui T, Yamashiro K, Kaji Y, Amano S, Ogura $Y$, et al. VEGF164-mediated inflammation is required for pathological, but not physiological, ischemia-induced retinal neovascularization. J Exp Med 2003; 198: 483-489.

19. $\mathrm{Ng}$ YS, Krilleke D, Shima DT. VEGF function in vascular pathogenesis. Exp Cell Res 2006; 312: 527-537.

20. Nader HB, Chavante SF, dos-Santos EA, Oliveira TW, dePaiva JF, Jeronimo SM, et al. Heparan sulfates and heparins: similar compounds performing the same functions in vertebrates and invertebrates? Braz J Med Biol Res 1999; 32: 529-538.

21. Regatieri CV, Dreyfuss JL, Melo GB, Lavinsky D, Farah ME, Nader HB. Dual role of intravitreous infliximab in experimental choroidal neovascularization: effect on the expression of sulfated glycosaminoglycans. Invest Ophthalmol Vis Sci 2009; 50: 5487-5494.

22. Sampaio L, Tersariol I, Lopes C, Bouças R, Nascimento F, Rocha $\mathrm{H}$, et al. Heparins and heparans sulfates. Structure, distribution and protein interactions. In: Verli H (Editor), Insights into carbohydrate structure and biological function. 1st edn. Kerala: Transworld Research Network; 2006. p 12-24.

23. Dreyfuss JL, Regatieri CV, Jarrouge TR, Cavalheiro RP, Sampaio LO, Nader HB. Heparan sulfate proteoglycans: structure, protein interactions and cell signaling. An Acad Bras Cienc 2009; 81: 409-429.

24. Alexopoulou AN, Multhaupt HA, Couchman JR. Syndecans in wound healing, inflammation and vascular biology. Int $J$
Biochem Cell Biol 2007; 39: 505-528.

25. Bass MD, Humphries MJ. Cytoplasmic interactions of syndecan-4 orchestrate adhesion receptor and growth factor receptor signalling. Biochem J 2002; 368: 1-15.

26. Beauvais DM, Rapraeger AC. Syndecans in tumor cell adhesion and signaling. Reprod Biol Endocrinol 2004; 2: 3.

27. Nunes SS, Outeiro-Bernstein MA, Juliano L, Vardiero F, Na$\operatorname{der} \mathrm{HB}$, Woods A, et al. Syndecan-4 contributes to endothelial tubulogenesis through interactions with two motifs inside the pro-angiogenic $\mathrm{N}$-terminal domain of thrombospondin-1. J Cell Physiol 2008; 214: 828-837.

28. Gallagher JT. Multiprotein signalling complexes: regional assembly on heparan sulphate. Biochem Soc Trans 2006; 34: 438-441.

29. Bix G, lozzo RV. Novel interactions of perlecan: unraveling perlecan's role in angiogenesis. Microsc Res Tech 2008; 71 : 339-348.

30. Knox SM, Whitelock JM. Perlecan: how does one molecule do so many things? Cell Mol Life Sci 2006; 63: 2435-2445.

31. Celie JW, Beelen RH, van den Born J. Heparan sulfate proteoglycans in extravasation: assisting leukocyte guidance. Front Biosci 2009; 14: 4932-4949.

32. Dolar-Szczasny J, Mackiewicz J, Bindewald A, Holz FG, Zagorski Z. [Fundus autofluorescence examination using a confocal scanning laser ophthalmoscope HRA (Heidelberg Retina Angiograph)]. Klin Oczna 2005; 107: 544-547.

33. Bland JM, Altman DG. Statistical methods for assessing agreement between two methods of clinical measurement. Lancet 1986; 1: 307-310.

34. Altman DG. Practical statistics for medical research. London: Chapman \& Hall; 1991.

35. Cordeiro MF, Guo L, Luong V, Harding G, Wang W, Jones $\mathrm{HE}$, et al. Real-time imaging of single nerve cell apoptosis in retinal neurodegeneration. Proc Natl Acad Sci U S A 2004; 101: 13352-13356.

36. Delori FC, Dorey CK, Staurenghi G, Arend O, Goger DG, Weiter JJ. In vivo fluorescence of the ocular fundus exhibits retinal pigment epithelium lipofuscin characteristics. Invest Ophthalmol Vis Sci 1995; 36: 718-729.

37. von Ruckmann A, Fitzke FW, Bird AC. Fundus autofluorescence in age-related macular disease imaged with a laser scanning ophthalmoscope. Invest Ophthalmol Vis Sci 1997; 38: 478-486.

38. Vaclavik V, Vujosevic S, Dandekar SS, Bunce C, Peto T, Bird AC. Autofluorescence imaging in age-related macular degeneration complicated by choroidal neovascularization: a prospective study. Ophthalmology 2008; 115: 342-346.

39. Bui TV, Han Y, Radu RA, Travis GH, Mata NL. Characterization of native retinal fluorophores involved in biosynthesis of $\mathrm{A} 2 \mathrm{E}$ and lipofuscin-associated retinopathies. $\mathrm{J} \mathrm{Biol}$ Chem 2006; 281: 18112-18119.

40. Jiang $X$, Couchman JR. Perlecan and tumor angiogenesis. J Histochem Cytochem 2003; 51: 1393-1410. 